Argentina, Brazil, Cuba, Chile and Uruguay (GASP Latin America). Most were observed in 2012 (12 isolates). After four unsuccessful attempts to implement national gonococcal antimicrobial surveillance, in 2016/2017 Brazil established a first national surveillance system with 550 isolates from seven sites across the country. Minimal inhibitory concentration (MIC), determined by agar dilution, revealed $42 \%$ to $79 \%$ of isolates resistant to ciprofloxacin, and $4 \%$ to $9.9 \%$ resistant to azithromycin, along with expected high rates of resistance to penicillin and tetracycline. Regarding resistance to ESCs, isolates with cefixime MIC bordering resistance (MIC $=0.125$ $\mathrm{mg} / \mathrm{L}$ ) were observed according to European cefixime resistance breakpoint, and were found in all five regions of Brazil (3.1 to 10.8\%). This result led to revision of the Brazilian Guidelines for STI Treatment; current recommendation is intramuscular (IM) ceftriaxone $500 \mathrm{mg}$ associated with azithromycin $1 \mathrm{~g}(\mathrm{VO})$ in a single dose. The rates of resistance have concerned Brazil's Ministry of Health and led to a new surveillance study including new collection sites (12), further epidemiological data, and samples from different anatomic sites, so as to monitor the spread of isolates and increase national representativeness. Challenges are to improve etiological diagnostics among PrEP users and PLHIV as well as in STI clinics; moreover, systematic surveillance of Neisseria gonorrhoeae antimicrobial resistance in Brazil and many Latin American countries is related to the organization of health systems, which do not require notification and often treat STI through a syndromic approach.

Disclosure No significant relationships.

\section{S19.3 PERSPECTIVES FROM THAILAND}

Pachara Sirivongrangson*. Thailand Ministry of Public Health, Department of Disease Control, Nonthaburi, Thailand

\subsection{6/sextrans-2019-sti.87}

Gonorrhea is a major public health concern in South East Asia including Thailand. In 2016, 9434 (14.3/100,000 population) of gonorrhea cases were reported. Complications including pelvic inflammatory disease, ectopic pregnancy and infertility compound the disease burden. Antimicrobial resistance (AMR) in Neisseria gonorrhoeae (NG) has been monitored. In addition, the novel Enhanced Gonococcal Antimicrobial Surveillance Program (EGASP) was initiated in 2015. Review the approach of public health on prevention, surveillance data and responses to the treat of AMR in NG including the EGASP in Thailand. This presentation will include the burden of gonorrhea, gonococcal antimicrobial susceptibilities to the drugs currently recommended for the treatment of gonorrhea (ceftriaxone, cefixime, and azithromycin), development of prevention strategies and responses to the threat of AMR gonorrhea and challenges. Public health approach on gonorrhea prevention is crucial and the threat of AMR in NG driven the urgent need to strengthen Gonococcal
Antimicrobial Surveillance Program and rapid response to treatment failure gonorrhea cases and control the spreading of AMR NG. Effective and combination prevention strategies at country and global level is needed.

Disclosure No significant relationships.

\section{S19.4 PUBLIC HEALTH APPROACHES TO GONORRHOEA PREVENTION AND THE THREAT OF ANTIBIOTIC RESISTANCE - PERSPECTIVES FROM EUROPE}

Otilia Mårdh*. European Centre for Disease Prevention and Control (ECDC), Solna, Sweden

\subsection{6/sextrans-2019-sti.88}

Background Gonorrhoea is the second most frequently reported STI in the European Union and European Economic Area (EU/EEA). Detection of multi- or extensively-drug resistant Neisseria gonorrhoeae isolates and cases of treatment failure to the first-line recommended therapy have been reported in the region. We present main epidemiological trends and implications for gonorrhoea prevention and control.

Methods The European Centre for Disease Prevention and Control (ECDC) collects data from EU/EEA countries on gonorrhoea diagnoses through the European Surveillance System and monitors antimicrobial resistance through the European Gonococcal Antimicrobial Surveillance Programme (EuroGASP). Annual ECDC reports on gonorrhoea epidemiology and gonococcal antimicrobial susceptibility aim to inform public health control efforts and evidence-based clinical guidelines in the EU/EEA. Most recent surveillance and Euro-GASP data are summarised.

Results In 2017, 89,239 gonorrhoea cases were reported by 27 EU/EEA countries, a 17\% increase compared to 2016. The EU/EEA notification rate was $22 / 100,000$ population, but national rates varied between 0.2 and 74.7 . Of cases with available information, $76 \%$ were males, 62\% aged 20-34 years, 52\% MSM, 24\% heterosexual men and 24\% women. HIV-positivity was $13.6 \%$ overall, $22.2 \%$ for MSM, $1.4 \%$ for heterosexual men and $0.6 \%$ for women. Between 2013-2017, gonorrhoea diagnoses increased by $67 \%, 31 \%$ and $13 \%$ among MSM, women and heterosexual men, respectively. The 2017 Euro-GASP data reported by 27 EU/EEA countries indicate stable levels of resistance to cefixime (1.9\% vs $2.1 \%)$, azithromycin (7.5 vs $7.5 \%)$ and ciprofloxacin (46.5\% vs $46.5 \%)$ and no resistance to ceftriaxone, compared to 2016.

Conclusions, recommendations Enhanced testing of populations at high-risk, appropriate treatment of cases and partner notification can facilitate prevention and control of gonorrhoea and should be optimally complemented with novel technology approaches adapted to deliver prevention interventions among MSM, a population that displays the highest burden and most accelerated increase in recent years. New therapeutic drugs and ideally a vaccine are needed.

Disclosure No significant relationships. 